\title{
Depth effect from a rotating line of constant length
}

\author{
MARIO ZANFORLIN and GIORGIO VALLORTIGARA \\ Università di Padova, Padua, Italy
}

\begin{abstract}
When a straight line is slowly rotated in the frontoparallel plane, subjects report seeing a compelling depth effect. The line appears slanted into 3-D space, with its end parts located at different distances from the observer and describing a sort of circular trajectory with respect to the frontal plane. As a result of this depth effect, the line appears longer than when it is located on the frontal plane, and the perceived length depends on the amount of tilt perceived. An explanation of this effect is presented, based on the hypothesis that the visual system minimizes differences in linear velocities in the 2-D velocity field. The results of an experiment in which subjects were required to evaluate the length of the tilting line were in close agreement with the expected values computed on the basis of the model. The results are discussed in relation to current theories of visual perception of structure from motion.
\end{abstract}

Stereokinetic effects occur when certain 2-D patterns are rotated in a plane perpendicular to the line of sight. For example, when a flat ellipse is slowly rotated in the frontoparallel plane, it appears first to deform with amoeba-like movements and then to tilt back and forth in 3-D space as a rigid circular disk (Musatti, 1924).

Various authors have claimed that such a stereokinetic phenomenon can be considered as an instance of the kinetic depth effect (see Tauber \& Kaufman, 1977; Wallach, Weisz, \& Adams, 1956). In the kinetic depth effect, contours of the shadow of a rotating 3-D object change in both orientation and length to produce a strong impression of depth (Wallach \& O'Connell, 1953). Obviously, in the stereokinetic effect there is not a simultaneous change in orientation and length, but only a rigid rotation of a flat pattern. However, it has been suggested that the motion of smooth curvilinear contours that lack identifiable features can be misperceived (Ullman, 1984b; Wilson, Robinson, \& Piggins, 1983). It seems that the visual system cannot detect the movement of a smooth elongated contour when the direction of movement is parallel to the contour itself (see also Movshon, Adelson, Gizzi, \& Newsome, 1983). In the case of a rotating ellipse, for example, one can imagine a partition of the actual motion into its orthogonal components: one component would be parallel to the contour (and not detected by retinal receptors), whereas the other would be perpendicular to the contour (and perhaps responsible for the amoeba-like transformations preceding the depth effect). Thus, although physically there is only a rigid, rotating flat pattern, the pattern of retinal stimulation may indeed be that of a shape continuously changing in both orienta-

This research was supported by C.N.R. Grant 86.00596 .04 . The authors' address is Dipartimento di Psicologia Generale, Universitá di Padova, Piazza Capitaniato 3, 35139 Padua, Italy. tion and length as in the case of the kinetic depth effect (for a similar argument, see Wallach et al., 1956).

Some years ago, however, Renvall (1929), Musatti (1955), and Mefferd and Wieland (1967) reported an interesting stereokinetic effect that occurs with a flat pattern that has no curvilinear contours at all. A straight white line or rod was slowly rotated on a black turntable; after some minutes of inspection, subjects reported seeing the rod slanted into 3-D space with its ends located at different distances, describing a sort of circular trajectory with respect to the frontoparallel plane. Similar observations have recently been confirmed and extended by Vallortigara, Bressan, and Zanforlin (1986) and Bressan and Vallortigara (1986). Note too that Johansson and Jansson (1968), during some studies on depth effects produced by a straight line's changing length and direction, discovered a similar phenomenon. They wrote:

The movement in depth for Stimulus Pattern 1 [which was identical to the pattern described by Renvall, 1929] was not predicted because this pattern has no change in length. At the present stage of the investigation we have to accept it, but it ought to be studied further. (p. 170)

Current algorithms for the extraction of structure from motion cannot account for the depth effect produced by such a pattern. All these algorithms start from a changing 2-D image and are primarily concerned with the problems of extracting a unique 3-D interpretation from such an ambiguous 2-D pattern (see Cutting, 1987; Johannson, von Hofsten, \& Jansson, 1980; Ullman, 1979, 1984a). Ullman (1984b) claimed that, in testing the role of the rigidity assumption, one must take care to ensure that two main requirements are met: (1) that 3-D cues are not available in the static projection; (2) that the motion is not misperceived. Since with a rotating rigid straight line there is neither misperception of movement due to the presence of smooth surfaces that lack identifiable fea- 
tures nor static 3-D cues, the only interpretation that these algorithms can extract is the "veridical" one: namely, a rigid, rotating figure that moves in the frontal plane, but not a figure moving in depth. Thus the mere existence of such a phenomenon constitutes a challenge for virtually all current theories of structure-from-motion perception.

Zanforlin (1987a, 1987b, in press) has recently proposed a new explanation for classic stereokinetic phenomema (such as Benussi's cone; see Musatti, 1924), which is based on a sort of minimization in the 2-D velocity field. In this paper we extend this model in order to explain the rotating-line stereokinetic effect as well.

\section{THE MODEL}

It is a fundamental principle of Gestalt theory that a process of minimization (or regularization) of the differences in the various aspects of the stimulus pattern that falls on the retina lies at the base of perceptual phenomena (Hatfield \& Epstein, 1985; Koffka, 1935).

Vector analysis as applied by Musatti (1928), Johansson (1950), Proffitt and Cutting (1980), and Cutting and Proffitt (1982) is a clear instance of the fruitfulness of the "minimum principle" in explaining perceptual phenomena (although obviously not all these authors are Gestalt psychologists).

To apply the minimum principle in our case, let us first consider the phenomenological aspects of a rectilinear segment drawn radially on a rotating disk, as shown in Figure 1. At first sight, the pattern is described by every subject as a rotating disk with a line drawn on it. After a few seconds of observation, however, if the disk surface is uniform, the disk appears motionless. The straight line appears to detach itself from the surface of the disk and slide on it, like a rotating clock hand. Metelli (1940), who first investigated this phenomenon, called it "phenomenal splitting"' between surface and object. Protracted observation produces a sequence of percepts: (1) the straight line appears to rotate around its own center while moving around center $Q$ of the disk; (2) the line appears slanted in depth at a well-defined angle of tilt and of a definite length. Note that at this stage the line appears longer than when it is located in the frontal plane. Usually the extremity of the line farther away from the center of rotation, $Q$, appears nearer to the observer. However, if the center of the line coincides with Q, either A or B may appear towards to the observer, depending on fixation or spontaneous alternation.

From a physical point of view, when the straight line rotates around $\mathrm{Q}$, in going from Position 1 to Position 2 (see Figure 1), the extremities $A$ and $B$ describe the arcs $\mathrm{AA}^{\prime}$ and $\mathrm{BB}^{\prime}$. Since all points of the line move around $\mathrm{Q}$ at the same angular velocity, their linear velocities will be different, depending on their distance from $Q$. The trajectory described by $\mathrm{O}$, the midpoint of the straight line, will be an arc of a circle that can be considered as the

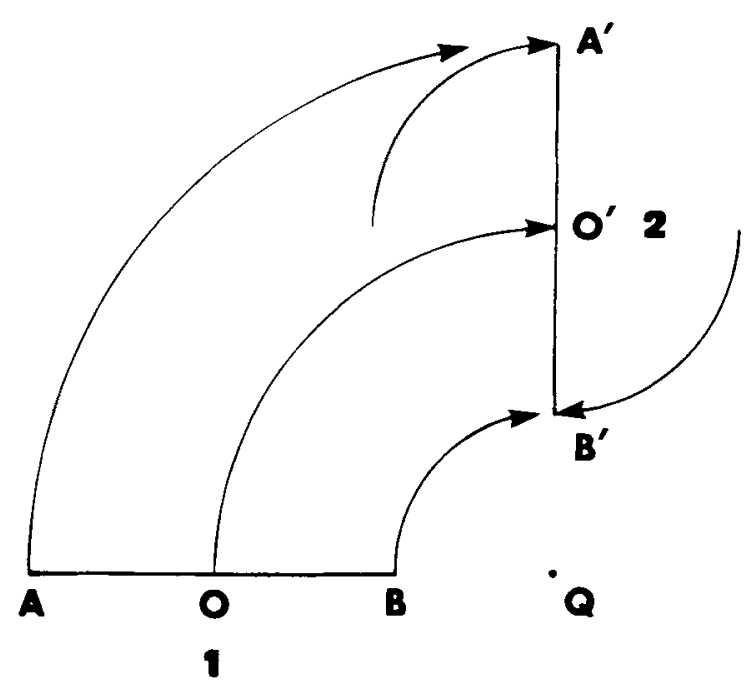

Figure 1. The segment $\mathrm{AB}$ is rotating around $\mathrm{Q}$. The two extreme points $A$ and $B$ appear to rotate around $O$ at equal velocities, while $O$ moves around $Q$ and describes a common component.

average length of the trajectories described by $A$ and $B$. Therefore, the linear velocity of $O$ can also be considered as the average of the linear velocities of $A$ and $B$. The linear velocity vectors of points $A$ and $B$ can be split into a common component, equal to that of $O$, and a residual component around $O$. The velocity of $A$ around $O$ will now appear equal (in scalar value) to that of $B$ around $\mathrm{O}$, with $\mathrm{O}$ being the center point that describes the common trajectory. It can be hypothesized, therefore, that a process of minimization, or zeroing, of the differences in linear velocities (in scalar values) between $A$ and $B$ explains the perceptual phenomenon described in (1) above, namely, that the straight line appears to rotate around its own center while moving around $Q$ (for similar considerations in explaining the rotating wheel phenomenon, see also Cutting \& Proffit, 1982; Duncker, 1929; Johannson, 1974; Musatti, 1928; Rubin, 1927).

At this point, the linear velocities (in scalar values) of $A$ and $B$ in relation to $O$ are equal. However, as $A$ and $B$ move around $O$, their linear velocities are different from that of $O$, whatever the velocity of $O$ may be. We therefore hypothesized a further process of minimization of the differences in linear velocities (in scalar values), which may well be performed simultaneously by the visual system. Repeating the previous reasoning, the average linear velocity between $A$ and $O$ is given by $A C, C$ being the midpoint between $A$ and $O$ (see Figure 2a). Since the physical angular velocities of all the points of the segment are the same, their relative linear velocities will depend on their relative distances. Hence the instantaneous apparent linear velocity $A$ in relation to $O$ will have a value proportional to the distance $\mathrm{AC}$ plus the distance $\mathrm{AO}$, that is, $A^{\prime} O$. The same reasoning can be repeated for $B$ in relation to $\mathrm{O}$ : its instantaneous linear velocity will be given 
(a)

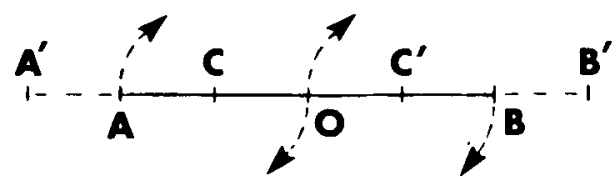

(b)

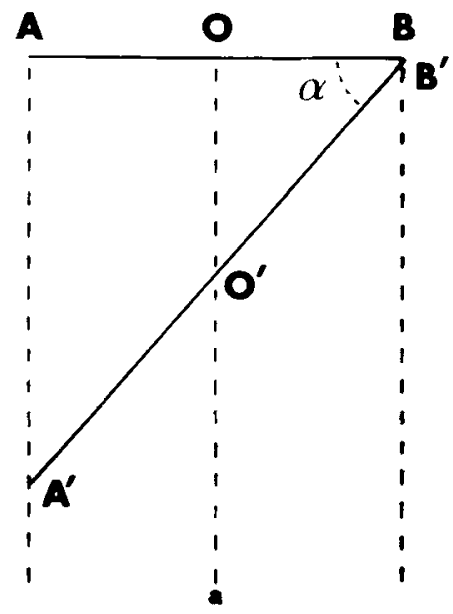

Figure 2. (a) The apparent length of the segment $A B$, as a consequence of the minimization of lincar velocities, will become $\mathbf{A}^{\prime} \mathbf{B}$ '. (b) View from above of the apparent displacement in depth of the segment $A B$ that becomes $A^{\prime} B^{\prime}$ ( $a=$ line of sight; $\alpha=$ angle of tilt with respect to the frontoparallel plane).

by $\mathrm{OB}^{\prime}$, that is, by $\mathrm{OB}+\mathrm{C}^{\prime} \mathrm{B}$ (see Figure $2 \mathrm{a}$ ). Note that in minimizing their velocity differences with respect to $O, A$ and $B$ cannot rotate around $C$ and $C^{\prime}$ because $O$ will have two equal linear velocities in opposite directions on the frontal plane. However, there is nothing to oppose a possible movement of the line around $\mathrm{O}$ in the third dimension, outside the frontal plane. Thus minimization of the differences in linear velocities can occur if the line is tilted in depth and the apparent distance $A^{\prime} B^{\prime}$ will be proportional to the minimized instantaneous linear velocities of the points. If this hypothesis is correct, then the straight line will appear (in depth) with a total length $A^{\prime} B^{\prime}$ $=A B+O B$ (where $\mathrm{OB}=\mathrm{AC}+\mathrm{OC}^{\prime}$ ), that is, $50 \%$ longer than when it appears in the plane. Suppose that the observer is located at a distance sufficient to approximate orthogonal projection; then the angle of tilt will be $\cos \alpha=\mathrm{AB} / \mathrm{A}^{\prime} \mathrm{B}^{\prime}$ (see Figure $2 \mathrm{~b}$ ). ${ }^{1}$

\section{EXPERIMENT 1}

We tested the theoretical model by requiring subjects to evaluate the length of a rotating line in different conditions.

\section{Methods}

Subjects. Four university students who were expert in viewing stereokinetic phenomena, but naive with regard to the predictions of our model, served as subjects.
Apparatus and Procedure. Three white line segments of different lengths $(4,6$, and $8 \mathrm{~cm})$ were rotated on a black turntable at a speed of $15 \mathrm{rpm}$. They were $0.5 \mathrm{~cm}$ wide. Each segment was placed according to one of the following conditions: (A) with its geometrical centers corresponding to the center of the turntable (extreme points with equal absolute physical velocities); (B) with one end placed on the center of the turntable (this point with zero absolute physical velocity); (C) with one end placed $2 \mathrm{~cm}$ away from the center of the turntable (extreme points with unbalanced absolute physical velocities). Because the hypothesis predicts that the apparent length of the line in depth does not depend on the absolute physical velocities, but rather on the relative velocities, we placed the lines in different positions on the turntable in order to test whether the absolute physical velocities of the lines' extremities had any effect on the apparent length of the line in depth.

The subjects were seated $2 \mathrm{~m}$ away from the figures, which rotated in the frontoparallel plane. The luminance of the figures was about $8 \mathrm{mfL}$ (the patterns were presented in reduced lighting conditions in order to minimize texture visibility of the turntable, while at the same time allowing the subjects to locate the turntable in the room). The subjects were required to evaluate the length of the slanted line (in centimeters) under three different conditions: (1) when the line was not moving; (2) when the subjects were not experiencing the depth effect (i.e., with the line rotating in the frontal plane); and (3) when experiencing the depth effect. The line lengths and judgment conditions were randomized for each subject, so as to reduce the likelihood of a response bias. To facilitate the task, a series of white lines of various lengths $(5,10,15$, and $20 \mathrm{~cm})$ were drawn on a sheet of black cardboard. The cardboard was located near the turntable and was clearly visible to the subjects. Viewing was monocular, in order to eliminate any stereoscopic cues present.

\section{Results}

The results are shown in Figure 3. A 3 (positions on the turntable) $\times 3$ (lengths of lines) $\times 3$ (judgment conditions) repeated measures analysis of variance was performed. The main effect of the position of the line on the turntable was not significant $[F(2,6)=2.761, p=.141]$. The main effect of the line length was significant $[F(2,6)=468.19, p<.001]$, as was the main effect of the judgment condition $[F(2,6)=27, p=.001]$. The position on the turntable $\times$ line length interaction was not significant $[F(4,12)=1.368, p=.302]$, nor was the position on the turntable $\times$ judgment condition interaction $[F(4,12)=2.267, p=.122]$. The line length $\times$ judgment condition interaction was significant $[F(4,12)=$ $3.751, p=.033$ ], whereas the position on the turntable $\times$ line length $\times$ judgment condition interaction was notsignificant $[F(8,24)=.561]$.

As can be seen, these results fit in well with the theoretically expected results for all values of line length (the expected values were, respectively, 6, 9, and $12 \mathrm{~cm}$ ). As expected, the position of the line on the disk did not affect the value of the perceived length. However, the position of the line with respect to the center of rotation affected the apparent location of the line with respect to the plane of the disk. When the center of the line was placed on the center of the disk (Condition A), all the subjects described the line as lying halfway through the plane of the disk, which appeared as a sort of "transparent," or 

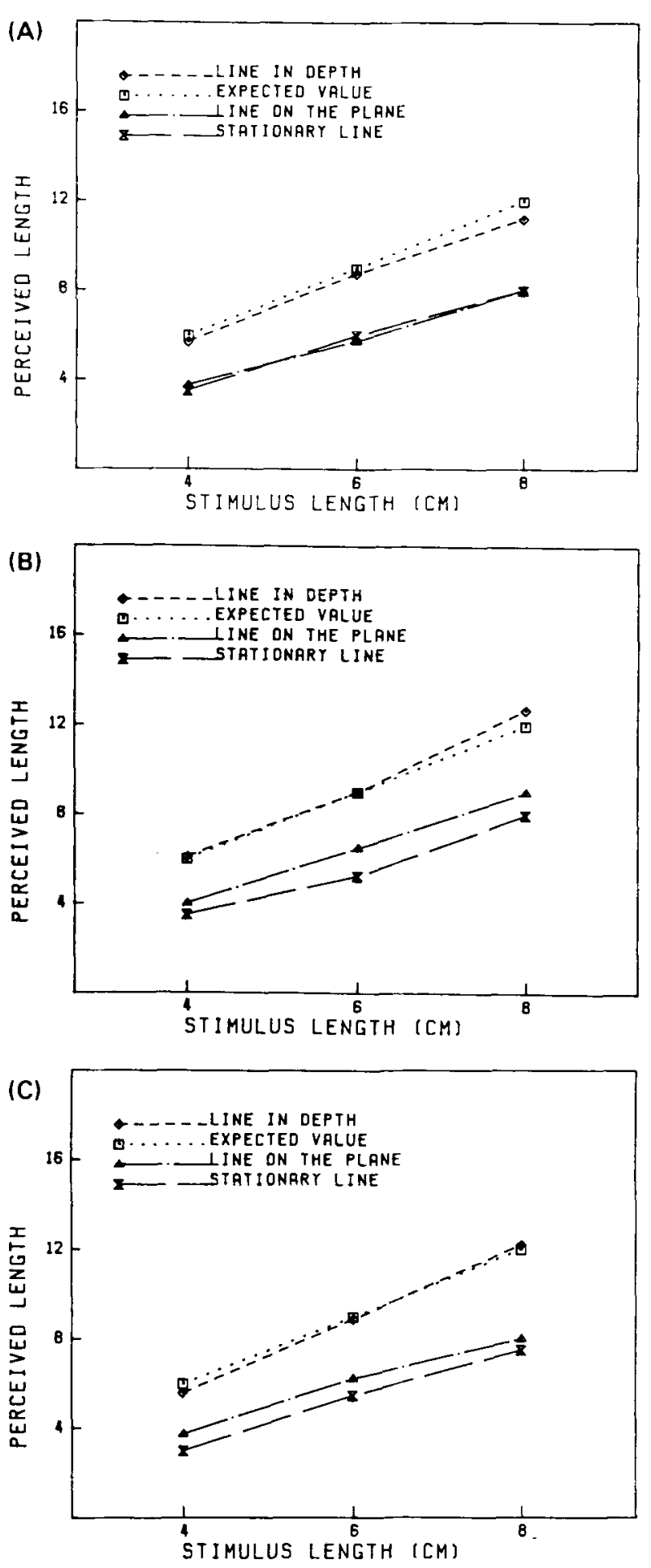

Figure 3. Apparent length judgments. Condition A: the center of the segment coincides with the center of the turntable; Condition B: one end of the segment coincides with the center of the turntable; Condition $\mathrm{C}$ : one end of the segment is placed $2 \mathrm{~cm}$ away from the center of the turntable. Average standard deviations were 1.26 for the line in depth, .64 for the line moving on the plane, .50 for the stationary line. In no case was there overlapping between the withdepth and without-depth estimations. dark empty space. One end of the line appeared to be closer to the observer and the other farther away; under prolonged observation, the end points could also reverse their position in depth (i.e., the line that was closest appeared to be farthest away, and vice versa). When one of the extreme points of the line coincided with the center of rotation of the disk (Condition B), or was placed $2 \mathrm{~cm}$ away from the center of the disk (Condition C), it acted as an anchor point and appeared to be lying on the plane of the disk, whereas the other extremity appeared to be nearer the observer. Depth inversion in this case was rather difficult even with considerable effort. We therefore concluded that: (1) the apparent length of the line does not depend on the absolute physical velocities of the extreme points but rather on the relative velocities, as predicted by the hypothesis; (2) the absolute physical velocities merely affect the apparent position of the line with respect to the plane of the disk.

\section{FURTHER ASPECTS OF THE HYPOTHESIS}

It seems paradoxical to increase the apparent distance (line length) between two points ( $A$ and $B$ ) in order to minimize differences in their linear velocities (in scalar values). It must be noted, however, that as a result of the minimization process, the apparent linear velocities of $\mathrm{A}$, $B$, and $O$ all become equal or averaged in relation to each other. This means that when the depth effect occurs, all points of the segment become apparently still or motionless in relation to each other. The situation is analogous to that of an object (the line segment considered as a whole unit) that does not rotate but revolves around an axis passing through $Q$ and continuously changes its tilt (or "nutates").

Although the rotation of the line lying on the frontal plane is not perceived as twisting, this is just the way in which the visual system interprets the situation. In fact, for a solid object, there will be a point inside the object at zero rotational velocity. In order to minimize the velocity difference between this point and all other points, as a result of the first minimization process the line is treated by the visual system as twisting during its rotation in the frontal plane. Then, when the line is shifted in depth, the twisting component can be eliminated during nutation: that is, the line moves in 3-D space by maintaining a stable spatial orientation. To understand this point, it may be useful to consider an analogy. The movement of the line in the frontal plane is analogous, for the perceptual system, to the movement of the index finger when, with the palm flat on the table, it is rotated so as to trace a circle with the tip of the finger. It is impossible to complete the circle unless the hand and finger are twisted. But if the hand is lifted from the table, there will be no difficulty in tracing a circle with the finger by keeping the hand steady and simply changing the tilt of the finger as it moves around. When the line is tilted in depth, it appears just as a finger tracing a circle with its tip: the whole finger will appear to trace a cone. 


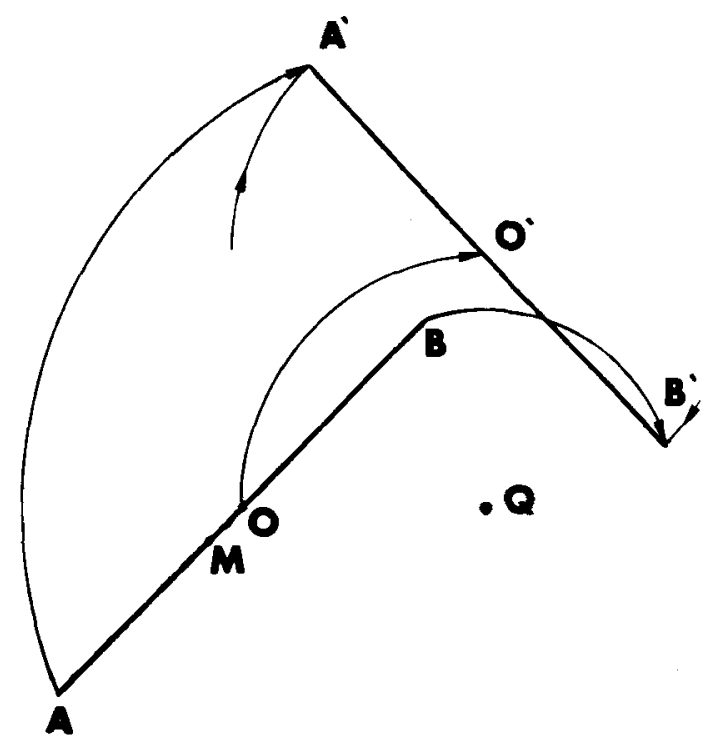

Figure 4. Rotation of $a$ line not colinear with center $Q$ of the disk. Subtraction of the common component described by $O$ leaves two unequal residual components relative to $A$ and $B$.

Now consider one point moving in relation to another steady point. In a similar situation, the velocity of the point will be $v=\theta d / t$ (where $d$ is the distance between the two points and $\theta$ the angle of displacement of the segment connecting the two points). For a given value of $v$, an increment of $d$ should be compensated by a reduced value of $\theta$, the angular displacement of the segment connecting the two points. Thus, from a psychological point of view, the apparent lengthening of the segment in depth will be accompanied by the impression that it moves at a reduced velocity. This is indeed what all the subjects reported: an impression of the slowing down of the segment when it was seen in depth.

\section{NONRADIAL LINES}

In Experiment 1, we considered a rotating line that was placed in a radial position with respect to the center of the disk. We think that this situation is particularly significant, because there is no reason, on the basis of current theories of structure-from-motion perception, for the line to appear slanted in depth. For a line in a nonradial position, however, there are other possible hypotheses that do predict a depth effect. These hypotheses, although not valid for a radial line, are still worth examining. Let us consider a rotating line placed at some angle with respect to the radial direction. If we apply the first process of minimization (i.e., that which explains the apparent rotation of the line around its own center), the line in Figure 4 will exhibit two main differences in comparison with a radial line. First, the difference in absolute (with respect to $Q$ ) linear velocity between the extreme points $A$ and $B$ will be smaller. Second, the point of average linear velocity (in relation to $Q$ ) will not correspond with the center (or centroid) of the line as it does with a radial line. The result is that if we subtract, as the common component, the trajectory described by the center $\mathrm{O}$, two unequal residual components relative to $\mathrm{A}$ and $\mathrm{B}$ will remain; that is, in this case the first minimization process is not possible on the frontal plane as it would be with the radial line. Thus it is possible that, in order to equalize the apparent velocity of $\mathbf{A}$ and $B$ around $O$, the line is shifted in depth in such a way that the unequal trajectories of $\mathrm{A}$ and $\mathrm{B}$ on the plane can become the projections in the frontal plane of equal trajectories in depth. An alternative possibility is that the visual system considers as the common component the trajectory of the point of average linear velocities. In this case the line might appear to rotate around M (see Figure 4). However, even this operation does not minimize linear velocity differences of $A$ and $B$ with respect to $M$, unless the line also modifies its apparent length. This will occur if the line appears tilted in depth in such a way as to equalize distances AM and BM. Thus nonradial lines may appear shifted in depth to allow the first minimization of velocity differences. As we have seen, for radial lines, the first minimization can occur in the frontal plane and a shift occurs as a consequence of the second minimization process. This is why nonradial lines are not a clean test of our hypothesis.

Because the apparent length of the nutating line in the second process of minimization depends only on the linear distance between the extreme points and the center $O$, whether the line is or is not in a radial position will not make any difference to its apparent length. A reduced difference in the absolute linear velocities of the extreme points (as in the case of nonradial lines) will merely produce the impression that the line moves in depth with reduced velocity. The main difference with respect to the case of a radial line would be its complicated apparent path. The nonradial line moving in depth as a result of the first minimization process will appear to change its apparent length continuously. This change in its apparent length may be perceived as a continuous depth displacement of the whole line; that is, the line may appear to approach the observer and recede across the plane of the rotating disk. Thus the apparent path of the line will be a composition of three different movements: one around its own center, another across the plane of the disk, and a third one around center $Q$. The second and third movements may appear combined in an elliptical path that cuts across the plane of the disk (see Figure 5).

Besides these complicated paths, there is another reason for supposing that with a nonradial line, the depth effect could be more unstable and difficult to obtain. Indeed, if an observer fixates one of the extreme points of a nonradial line, its retinal projection will be identical to that of a radial line. Thus we will have a nonradial line with respect to the frame of reference of the environment, and a radial line with respect to the retinal frame of reference. This situation is likely to cause unstable and conflicting perceptions. 


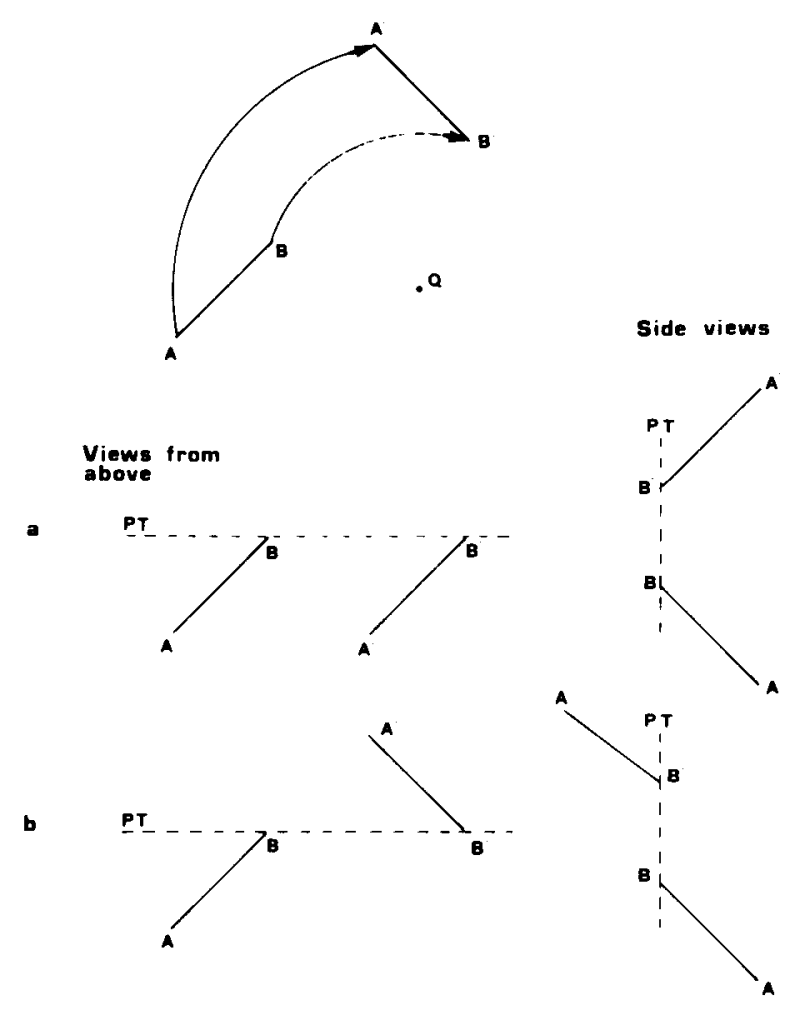

Figure 5. Schematic representation of the paths described by a nonradial line. PT represents the plane of the turntable, $a$ and $b$ represent alternative paths in 3-D space.

\section{EXPERIMENT 2}

\section{Methods}

Subjects. Four subjects who were expert in viewing stereokinetic phenomena but naive as regards our hypotheses were used as observers.

Apparatus and Procedure. The apparatus and general procedure were identical to those in Experiment 1. The subjects were required to compare (1) the perceptions elicited by nonradial lines placed at $45^{\circ}$ and $90^{\circ}$ relative to the radial position with (2) radial lines of the same length $(6 \mathrm{~cm})$. The conditions of presentation were identical to those in Experiment 1. Since we noticed during preliminary and informal observations that very similar depth effects could be obtained by substituting the lines with configurations of only two white dots (corresponding to the extreme points of the line), we asked our subjects to evaluate patterns composed of only two dots corresponding to the extremities of the line in the various conditions (i.e., virtual lines placed in a radial or nonradial position).

\section{Results}

As expected, all the subjects reported that with nonradial lines, more observation was required in order to achieve the depth effect. Moreover, the effect was less stable and compelling than it was with radial lines. One reason was that the nonradial lines described a very complex trajectory in 3-D space: they appeared to rotate in depth around their own center while rotating around the center of the disk. Thus they also appeared to change their apparent length and position in depth. The subjects also reported that it was easier to achieve a more stable depth effect by tracking one of the extreme points rather than fixating on the center of the line. However, when the subjects were required to compare the lengths of the lines in depth, they reported no significant differences between line lengths in radial and nonradial positions: the average values were $9.62(S D=.47)$ for the nonradial line at $45^{\circ}, 8.25(S D=1.32)$ for the nonradial line at $90^{\circ}$, and $8.75(S D=.95)$ for the radial line $[F(2,6)=1.461$, $p=.304]$. Interestingly, identical results were obtained by substituting whole lines with patterns consisting of only two white dots. The subjects reported seeing the movement in depth as that of a virtual line connecting the two points; the distance between the two points (in depth) was judged identical to that of a complete line of the same objective length: the average value was $9.6(S D=.80)$. It is worth noting that $3-\mathrm{D}$ perceptions elicited by two-light configurations mounted on a rolling wheel have also been described by Cutting and Proffitt (1982), although their study was not concerned with the present problem. From a phenomenological point of view, it is interesting that when the depth effect occurred, the region between the two points appeared to have surface-like characteristics (i.e., a sort of phantom object was visible, although there were no luminance differences in the stimulation). This intriguing effect deserves further investigation.

\section{GENERAL DISCUSSION}

Mefferd and Wieland (1967) suggested that verticalhorizontal anisotropies of the visual field can account for the effect produced by the rotating line. They claimed that during rotation, the line segment appears to lengthen when passing through the vertical axis and to shorten when passing through the horizontal axis. The depth effect, therefore, should be obtained in order to eliminate apparent deformations in the length of the line. Empirical results, however (see Künnapas, 1957), showed that the magnitude of the vertical-horizontal illusion was about $7 \%$. Our results showed that the apparent lengthening of the line in depth was $50 \%$ of the objective length of the straight line: a value too high to be explained by resorting to the vertical-horizontal illusion.

Current algorithms for the extraction of structure from motion are based on some sort of constraint that permits the resolution of the problem of uniqueness by 3-D interpretations. The constraint used most is the so-called rigidity assumption (see Cutting, 1987; Ullman, 1979, 1984a). Although the rigidity assumption has proved useful in machine vision, it is possible that the human visual system relies on entirely different principles (see also Ullman, 1986). Whether the rigidity assumption has any psychological validity is still an open question, about which several authors have expressed serious doubts (see, e.g., Bressan \& Vallortigara, 1987; Braunstein \& Andersen, 1984; Pomerantz, 1983; Schwarz \& Sperling, 1983). It is evident that the rigidity assumption is totally inadequate for explaining the rotating line stereokinetic effect: 
if a rigid pattern is already visible in the frontal plane, why should it shift in depth?

Our hypothesis-that the visual system minimizes differences in linear velocities-proved useful in explaining stereokinetic phenomena (see also Zanforlin, 1987a, 1987b), allowing quantitative predictions; it may be considered a valid alternative approach to the general problem of structure-from-motion perception.

\section{REFERENCES}

Braunstejn, M. L., ANDersen, G. J. (1984). A counterexample to the rigidity assumption in the visual perception of structure-frommotion. Perception, 13, 213-217.

Bressan, P., Vallortigara, G. (1986). Multiple 3-D interpretations in a classic stereokinetic effect. Perception, 15, 405-408.

Bressan P., \& Vallortigara, G. (1987). Learning to see stereokinetic effects. Perception, 16, 187-192.

CutTing, J. E. (1987). Perception and information. Annual Review of Psychology, 38, 61-90.

Cutting, J. E., \& ProffitT, D. R. (1982). The minimum principle and the perception of absolute, common and relative motions. Cognitive Psychology, 14, 211-246.

DuNCKer, K. (1929). Über induzierte Bewegung. Ein Beitrag zur Theorie optischen wahrgenommer Bewegung. Psychologische Forschung, 12, $159-180$.

Hatfield, G., \& Epstein, W. (1985). The status of the minimum principle in the theoretical analysis of visual perception. Psychological Bulletin, 97, 155-186.

Johansson, G. (1950). Configurations in event perception. Uppsala, Sweden: Almqvist \& Wiksell.

JoHANsson, G. (1974). Vector analysis in visual perception of rolling motion: A quantitative approach. Psychologische Forschung, 36, 311-319.

Johansson, G., \& JAnsson, G. (1968). Perceived rotary motion from changes in a straight line. Perception \& Psychophysics, 4, 165-170.

Johansson, G., von Hofsten, C., \& JANSson, G. (1980). Event perception. Annual Review of Psychology, 31, 27-63.

KoFFKA, K. (1935). Principles of Gestalt psychology. New York: Harcourn Brace.

KüNNAPAs, T. M. (1957). The vertical-horizontal illusion and the visual field. Journal of Experimental Psychology, 53, 405-407.

MefFerd, R. B., \& Wieland, B. A. (1967). Perception of depth in rotating objects: 1 . Stereokinesis and the vertical-horizontal illusion. Perceptual \& Motor Skills, 25, 93-100.

Metelu, F. (1940). Ricerche sperimentali sulla percezione del movimento. Rivista di Psicologia, 36, 319-370.

Movshon, J. A., Adelson, E. H., Gizzi, M. S., \& Newsome, W. T. (1983). The analysis of moving visual patterns. In C. Chagras, R. Gattas, \& C. Gross (Eds.), Pattern recognition mechanisms (pp. 117-151). Rome: Pontificia Accademia Scientarum.

Musatti, C. L. (1924). Sui fenomeni stereocinetici. Archivio Italiano di Psicologia, 3, 105-120.

MusatT, C. L. (1928). Sui movimenti apparenti dovuti ad illusione di identità di figura. Archivio di Psicologia, 6, 205-219.
Musatti, C. L. (1955), La stereocinesi e la struttura dello spazio visibile. Rivista di Psicologia, 49, 3-57.

Pomerantz, J. R. (1983). The nubber pencil illusion. Perception \& Psychophysics, 33, 365-368.

Proffit, D. R., Cutring, J. E. (1980). An invariant for wheelgenerated motions and the logic of its determination. Perception, 9, 435-449.

Renvall, P. (1929). Zur theorie des stereokinetischen Phänomens. Annales Universitatis Aboensis, Series B, 10, 13-75.

Rubin, E. (1927). Visuelle wahrgenommene wirkliche Bewegungen. Zeitschrifi für Psychologie, 103, 384-392.

Schwartz, B. J., \& Spring, G. (1983). Luminance controls the perceived 3-D structure of dynamic 2-D displays. Bulletin of the Psychonomic Society, 21, 456-458.

TAUBer, E. S., KaUfman, L. (1977). Fixation and the stereokinetic phenomenon. Perception \& Psychophysics, 22, 241-244.

Ullman, S. (1979). The interpretation of visual motion. Cambridge, MA: MIT Press.

UllmaN, S. (1984a). Maximizing rigidity: The incremental recovery of 3-D structure from rigid and non-rigid motion. Perception, 13, 255-274.

Ullman, S. (1984b). Rigidity and misperceived motion. Perception, 13, 218-219.

Ullman, S. (1986). Competence, performance and the rigidity assumption. Perception, 15, 644-646.

Vallortigara, G., Bressan, P., \& Zanforlin, M. (1986). The Saturn illusion: A new stereokinetic effect. Vision Research, 26, 811-813.

WAllach, H. , O'CONNELL, D. N. (1953). The kinetic depth effect. Joumal of Experimental Psychology, 45, 205-217.

Wallach, H., Weisz, A., \& Adams, P. A. (1956). Circles and derived figures in rotation. American Journal of Psychology, 69, 48-59.

Wilson, J. A., Robinson, J. O., \& Piggins, D. S. (1983). Wobble cones and wobble holes: The stereokinetic effect revisited. Perception, 12, 187-193.

ZANFORLIN, M. (1977a). La determinazione teorica dell'altezza del cono stereocinetico di Musatti. Atti e Memorie dell'Academia Patavina di Scienze, Lettere ed Arti, 99, 33-46.

Zanforlin, M. (1987b). The height of Musatti's stereokinetic cone. Fourth Intermational Conference on Event Perception and Action. August 24-28, 1987, Trieste.

ZANforlin, M. (in press). The height of a stereokinetic cone: A quantitative determination of a 3D effect from 2D moving patterns without a "rigidity assumption." Psychological Research.

\section{NOTE}

1. One may ask why the minimization process stops at this point. Consider that the first minimization occurs on the 2-D plane, and that the second minimization process requires the line to be shifted in 3-D space: further minimizations would require shifts of the line into the 4th, 5 th...nth dimensions.

(Manuscript received June 1, 1987; revision accepted for publication April 11, 1988.) 